\title{
Formación Docente Socio Comunitaria en la Carrera de Educación Inicial de la Universidad Nacional de Educación
}

\author{
Autoras: Ormary Egleé Barberi Ruiz \\ Universidad Nacional de Educación, UNAE \\ ormary.barberi@unae.edu.ec \\ Azogues, Ecuador \\ https://orcid.org/0000-0002-3628-3677 \\ Gisselle Margarita Tur Porres \\ Universidad Nacional de Educación, UNAE \\ gisselle.tur@unae.edu.ec \\ Azogues, Ecuador \\ https://orcid.org/0000-0003-1494-0549
}

\section{Resumen}

La carrera de Educación Inicial de la Universidad Nacional de Educación replantea la formación de docentes-investigadores en el contexto ecuatoriano y regional, que facilite procesos de enseñanza-aprendizaje con niños/as de 0 a 5 años, como actores de transformación en territorio mediante la atención y resolución de problemas en el ámbito educativo, desde un marco de equidad, interculturalidad, inclusión, innovación pedagógica y social. En este sentido, se presenta un análisis de la carrera en su dimensión sociocomunitaria, según los fundamentos pedagógicos de la universidad, elementos curriculares y lo que demanda el territorio respectivo. La metodología empleada se enmarca en el enfoque cualitativo y en la investigación documental. Los aportes teóricos son fundamentados en la Gestión Social del Conocimiento y las concepciones de vinculación con la colectividad según Vallaeys (2014), Martínez (2011) y Santos (2004). La revisión documental se realizó desde la dimensión política, educativa y social, revelada además en informes institucionales. A partir del análisis fue posible lograr la fundamentación conceptual y contextual de la formación docente en la carrera de Educación Inicial desde y para una dimensión socio comunitaria; su caracterización y los avances y desafíos de este proceso con énfasis en las prácticas preprofesionales como escenario dinamizador.

Palabras clave: formación de docentes; enseñanza superior; práctica pedagógica; responsabilidad social.

Fecha de Recepción: 29-09-2018

Fecha de Aceptación: 15-12-2018 


\title{
Socio-Community Teacher Training in the Early Childhood Education Career at the National University of Education
}

\begin{abstract}
The Initial Education career of the National University of Education reconsiders the training of teachers-researchers in the Ecuadorian and regional context, which facilitates teaching-learning processes with children from 0 to 5 years old, as actors of transformation in territory through the attention and resolution of problems in the educational field, from a framework of equity, interculturality, inclusion, pedagogical and social innovation. In this sense, an analysis of the career in its socio-community dimension is presented, according to the pedagogical foundations of the university, curricular elements and what the respective territory demands. The methodology used is part of the qualitative approach and documentary research. The theoretical contributions are based on the Social Management of Knowledge and the conceptions of connection with the collectivity according to Vallaeys (2014), Martínez (2011) and Santos (2004). The documentary review was carried out from the political, educational and social dimension, also revealed in institutional reports. From the analysis, it was possible to achieve the conceptual and contextual foundation of teacher training in the Initial Education career from and for a community-based dimension; its characterization and the advances and challenges of this process with emphasis on preprofessional practices as a dynamic scenario.
\end{abstract}

Keywords: teacher education; higher education; teaching practice; social responsibility.

Date Received: 29-09-2018

Date Acceptance: 15-12-2018 


\section{Introducción}

Los desafíos de las Instituciones de Educación Superior (IES) son cada vez más exigentes, dadas las circunstancias de la sociedad actual en cuanto a la formación tecnológica, profesional e investigativa de sus miembros en consonancia a las necesidades y demandas de los territorios en el marco de un enfoque sostenible. En consecuencia, se debe revisar y replantear la Responsabilidad Social Universitaria (RSU), como condición de las instituciones para contribuir en la sostenibilidad de los procesos que debe transitar de la declaración explícita y filantrópica, como parte de sus aspiraciones institucionales, contenidas en sus protocolos administrativos para la concreción de transformaciones requeridas en contextos reales, sobre la base de relaciones "simbióticas" entre los actores y agencias a favor del bienestar común, conservación de la naturaleza y las culturas.

En este orden de ideas, se asume lo planteado por Vallaeys (2014): en cuanto al sentido holístico, integrador y ético de la Responsabilidad Social (RS), su desarrollo permite a la organización un sentido de "conciencia de sí misma, de su entorno, y de su papel en su entorno, en una articulación pocas veces practicada entre ética y eficacia" (pág. 3). Las relaciones entre la institución u organización deben atender al bienestar de las partes involucradas, a la ayuda mutua, al diálogo y al consenso permanente.

La formación docente profesional que se imparte en las diversas IES latinoamericanas, tiene la misión de propiciar una gestión universitaria con compromiso social que derive en pertinencia académica, científica y social, según los perfiles de sus egresados y en correspondencia a la sociedad actual. En tal sentido, es necesario minimizar la brecha entre el conocimiento científico y la demanda del territorio, según Santos (2004): es el "tránsito del conocimiento universitario al conocimiento pluriuniversitario" (pág. 25). Contextualizar los conocimientos científicos, mediados por las particularidades de los contextos, constituye una condición sine qua non para redimensionar la 
docencia, la investigación y una vinculación universitaria pertinente.

Las argumentaciones señaladas procuran de una educación superior que facilite procesos de formación que emancipen a los actores socioeducativos desde y para un accionar docente transformador de los escenarios socio-educativos en el marco de los Objetivos de Desarrollo Sostenible (ODS), contenidos en el Programa de las Naciones Unidas para el Desarrollo (PNUD, 2016, pág. 1); como parte criterios que caracterizan la calidad educativa en consonancia al desarrollo educativo, socio-cultural y económico actual.

La Universidad Nacional de Educación (UNAE, 2016a), como centro de formación docente comprometido con la transformación de Ecuador y de la región, en consonancia con sus fundamentos de creación, considera el principio de pertinencia para que sus programas de formación "respondan a las expectativas y necesidades de la sociedad, a la planificación nacional, al régimen de desarrollo, a la prospectiva de desarrollo científico, humanístico y tecnológico mundial y a la diversidad cultural" (pág. 7).

Es de particular importancia y un desafío permanente que la carrera de Educación Inicial en la UNAE contribuya en la formación de docentes investigadores con compromiso social para que diseñen y dirijan procesos de enseñanza y aprendizaje de calidad en niños y niñas entre 0 y 5 años; representen a actores de transformación en territorio, mediante la atención y resolución de problemas en el ámbito de la Educación Inicial sobre la base de la vinculación de los contextos educativos (centro infantil, familia y comunidad), la equidad, la interculturalidad, la inclusión y la innovación pedagógica y social.

\section{Metodología}

A partir de los argumentos señalados anteriormente y su contextualización al ámbito de la UNAE, surge el interés por la temática de análisis ante la necesaria revisión, construcción y re-construcción constante del hacer educativo que deben asumir los actores docentes de la carrera de 
Educación Inicial en la UNAE, sobre la base de la interacción de las actividades de docencia, investigación y vinculación con la sociedad, facilitando procesos de observación permanente y experiencias en la práctica educativa, enmarcados en este caso en fundamentos y principios de una educación de calidad sostenible y de responsabilidad social universitaria (RSU).

El objetivo del presente escrito consiste en presentar un análisis de la formación docente en la carrera de Educación Inicial de la UNAE en su dimensión socio-comunitaria, según los fundamentos pedagógicos y curriculares de la UNAE; además de las particularidades del contexto, específicamente en el escenario de las prácticas preprofesionales. En tal sentido, el análisis está centrado en fundamentar y caracterizar la formación docente en el contexto de la UNAE, desde y para un enfoque sociocomunitario; así también, los avances y desafíos respectivos.

Para activar la construcción del análisis fue necesario partir de la siguiente interrogante: ¿Cómo repensar el proceso formativo del docenteinvestigador en la carrera de Educación Inicial de la UNAE en concordancia con los contextos sociales y educativos que inciden en la infancia?.

La construcción de las respuestas a las interrogantes planteadas requirió la metodología que se enmarca en el enfoque cualitativo y en la investigación documental y empírica en relación a los aportes teóricos en cuanto a la Gestión Social del Conocimiento y las concepciones de vinculación con la colectividad desarrolladas por Vallaeys, Martínez y Santos; así como también, la revisión de fuentes internas de la UNAE (estatutos, proyecto de carrera y microcurrículo respectivo, modelos pedagógico, prácticas preprofesionales y de vinculación con la sociedad, entre otros) y fuentes externas referidas a la normativa educativa del contexto.

La revisión permitió propiciar procesos de indagación, análisis y discusión acerca del carácter socio comunitario de la formación docente en la 
UNAE y derivar en los siguientes resultados: a). fundamentación conceptual y contextual de la formación docente en la carrera de Educación Inicial desde y para una dimensión socio comunitaria; b). caracterización de la dimensión pedagógica-curricular de la carrera de Educación Inicial, la vinculación con la colectividad y la formación docente socio comunitaria; y c). precisar los avances y desafíos de este proceso de formación en la carrera de El de la UNAE, según lo que demanda el territorio respectivo a partir de las prácticas preprofesionales como escenario dinamizador.

\section{Resultados del análisis}

\subsection{Transformar la educación con compromiso social}

La UNAE requiere mantenerse en una revisión, construcción y reconstrucción permanente de su hacer académico, científico y tecnológico en el marco de una educación de calidad sostenible. En tal sentido, este texto es motivo de encuentro para proponer y generar nuevas acciones que continúen contribuyendo a garantizar una educación equitativa y de calidad, que transforme las políticas y las prácticas educativas de manera activa y participativa con la comunidad. Tal como se menciona en el texto de Herdoiza (2015), Construyendo igualdad en la educación superior:

La educación superior está llamada a formar profesionales conscientes, críticos y comprometidos con la sociedad (...) a poner fin a la reproducción de prácticas de discriminación, inequidad y exclusión, y a construir una sociedad más igualitaria y justa. La educación superior del Ecuador asume este reto e, igualmente, reitera su compromiso de seguir impulsando el paradigma biocéntrico de desarrollo (pág. 9).

Por otra parte, la Constitución de la República del Ecuador, aprobada en referéndum por el pueblo ecuatoriano en la Asamblea Nacional Constituyente (2008), en su apartado educativo, sección quinta, asegura que:

La educación se centrará en el ser humano y garantizará su 
desarrollo holístico, en el marco del respeto a los derechos humanos, al medio ambiente sustentable y a la democracia; será participativa, obligatoria, intercultural, democrática, inclusiva y diversa, de calidad y calidez; impulsará la calidad de género, la justicia, la calidad y la paz; estimulará el sentido crítico, el arte y la cultura física; la iniciativa individual y comunitaria, y el desarrollo de competencias y habilidades para crear y trabajar (art. 27).

Amparados en los derechos garantizados por la Constitución de la República y organismos del Estado, la UNAE potencia una formación inicial holística, promueve la formación de docentes-investigadores, piensa a los sujetos educativos como actores de construcción y reflexión en el proceso de aprendizaje, y concibe a la niña y al niño como sujeto de derecho. Es así como la UNAE asume esa responsabilidad en la formación de docentesinvestigadores con compromiso ético y responsabilidad social. Este compromiso, a su vez, nos conduce a cuestionar aquellos pensamientos hegemónicos que nos lleven a asimilar a todos los sujetos, colectivos, comunidades desde una sola mirada, creando espacios de construcción del conocimiento con la participación de todos los actores educativos.

La UNAE es precursora de la transformación cultural y educativa del país, sentando precedentes innovadores en Ecuador y en la región, en el proceso de formación inicial docente. Asimismo, la universidad proporciona condiciones óptimas en los procesos de enseñanza-aprendizaje, centrándose en políticas educativas y prácticas pedagógicas emancipadoras que privilegian la equidad y la inclusión, sustentadas en un modelo pedagógico de la UNAE (2017a): "que estimula el pensamiento crítico, las competencias investigativas y la vinculación con la colectividad desde donde construir comunidad de aprendizaje" (pág. 24). En este sentido, la UNAE se consolida como un agente cultural que privilegia una formación socio-humanista e integra a todos los actores educativos de manera inclusiva y equitativa. 
En este marco, la carrera de Educación Inicial se inscribe en una propuesta pedagógica innovadora, que potencia la formación del docenteinvestigador con un componente importante de vinculación con la comunidad. La relación de la trilogía docencia-investigación y vinculación con la colectividad, en criterio de las autoras representa el elemento rector que privilegia el carácter socio-comunitario de la formación teórica, metodológica y práctica del estudiante. La carrera en referencia cumple un rol central en la formación académica integral desde el proceso reflexivo de la experiencia.

Por otro lado, pensar la interrelación: familia- escuela y comunidad, caracteriza la formación del docente-investigador de Educación Inicial en UNAE; que piensa la niñez y la comunidad en contexto, desde una perspectiva histórica, social y cultural, para una educación de calidad, equitativa e inclusiva. Es por lo que, el vínculo responsable con la niñez, la familia, los docentes y la comunidad deber ser fortalecido en cada ciclo de formación, como eje de transversalización, y que tribute a acciones de cambio, desde un pensamiento crítico y liberador para transformar la educación ecuatoriana.

En particular, la reflexión señalada permite apelar al diálogo y reflexión conjunta desde un enfoque inclusivo y de relaciones interculturales, en relación con la comunidad, asumiendo que la vinculación universitaria debe generar impactos educativos y socio-comunitarios en ambas partes del territorio, la formación requerida en la carrera de Educación Inicial para modelar el pensamiento teórico y práctico de sus aprendices en los diversos ciclos y los contextos socio-educativos con los cuales se establecen alianzas estratégicas y convenios en mutuo acuerdo para las transformaciones socio-educativas.

Ante este escenario dinámico de construcción y re-construcción, la vinculación universitaria de la UNAE debe generar impactos diversos y en beneficio de los actores y agencias involucradas, constituyendo las prácticas preprofesionales de las IES en escenario sustancial para dichos procesos. En consonancia con el planteamiento de Martínez (2011): "responsabilidad social 
universitaria constituye una estrategia de gestión que propicia un enfoque holístico sobre la propia organización universitaria, concibiendo iniciativas interdisciplinarias" (pág. 2).

Es significativo mencionar que en el contexto ecuatoriano las prácticas preprofesionales constituyen según el Consejo de Educación Superior (CES, 2016) “...actividades de aprendizaje orientadas a la aplicación de conocimientos y al desarrollo de destrezas y habilidades específicas que un estudiante debe adquirir para un adecuado desempeño en su futura profesión" (pág. 42). Los procesos de formación de la práctica propician una sinergia para la modelación de la actuación preprofesional del aprendiz que demanda la concreción de conocimientos, actitudes, habilidades, valores y emociones, que se conjugan en los escenarios socio-educativos reales para responder a la atención y solución de situaciones, casos y problemas socio-educativos.

Así también, la UNAE (2018), concibe las prácticas como "un entorno privilegiado para el aprendizaje y mejora de la práctica docente, tanto de estudiantes y docentes de la UNAE como de directivos y docentes de las escuelas participantes" (pág. 2). La dinámica que se genera en la praxis preprofesional propicia ajustes y cambios en la calidad educativa de las instituciones educativas involucradas sobre la base de los principios que guían el modelo pedagógico de la UNAE, como guía fundamental en la construcción del proceso de formación, para generar y provocar aprendizajes relevantes. Son principios rectores del modelo: aprender haciendo; esencializar el currículo; currículum basado en casos, problemas y proyectos; promover la didáctica invertida, el "flipped classroom"; las redes sociales virtuales y las plataformas digitales; primar la cooperación y fomentar el clima de confianza; fomentar la metacognición; apostar decididamente por la evaluación formativa; estimular la función tutorial del docente; potenciar de forma decidida la interculturalidad.

Tales principios son congruentes con las competencias básicas del 
docente-investigador que se forma en la UNAE, y a la vez acompañan el proceso de crear, practicar y reflexionar el modelo curricular de la universidad, en la acción de diseñar, comprender, actuar y evaluar de una forma coherente al proyecto educativo de la UNAE.

El diálogo que se genera a partir de la integración de saberes, guiados por un núcleo problémico y ejes que transversalizan los ciclos, proporcionan condiciones de gran valor pedagógico para privilegiar el sentido de la formación inicial en un escenario social cambiante y de incertidumbre que permea el escenario de la práctica preprofesional en contextos educativos específicos. Es por lo que, a partir de este panorama de formación docente, constituye un gran desafío para la El potenciar las relaciones de los docentes en vínculo con la niñez, la familia y la comunidad, que promueva la escucha y participación activa de la comunidad educativa.

\subsection{Redimensionamiento socio-comunitario de la formación docente en la carrera de Educación Inicial}

En el marco de la revisión curricular de la carrera a partir del modelo pedagógico y el de prácticas preprofesionales se puede afirmar la trascendencia de los elementos del microcurrículo de Educación Inicial en la dimensión socio-comunitaria, el núcleo problémico y el eje integrador transversalizan la dinámica de formación de cada ciclo en relación al sentido de los campos de formación: básica, profesional y de titulación, es decir, proporcionan condiciones curriculares inter y transdisciplinarias para la modelación de la formación pedagógica e investigativa con compromiso y pertinencia social, en cuanto a los escenarios de aprendizaje. 
En tal sentido, los escenarios de formación docente en la carrera de Educación Inicial de la UNAE se caracterizan por:

- Actividades de docencia basada en la tutorización docente en las diferentes asignaturas de cada ciclo académico, prácticas y en la construcción de propuestas de solución ante casos, situaciones o problemas en escenarios educativos y socio-comunitarios específicos.

- El desarrollo de la práctica preprofesional en contextos educativos específicos de la Educación Inicial ecuatoriana y en correspondencia a las particularidades del contexto; compartiendo escenarios sociocomunitarios particulares según las características del territorio.

- Énfasis en el desarrollo de competencias para la atención y construcción de soluciones a situaciones, casos y problemas educativos (ciclo a ciclo), mediante los proyectos integradores de saberes (PIENSA) que los estudiantes realizan con pertinencia social según el acompañamiento de sus tutores académicos y de asignatura en escenarios educativos. EI PIENSA constituye un dispositivo pedagógico y didáctico para el desarrollo de competencias investigativas en escenarios profesionales.

- La organización curricular a partir de los elementos orientadores de la formación docente, el eje transversal y nudo problémico deben interactuar en la dinámica de formación docente. En la gráfica 1, se visualizan los elementos curriculares para cada uno de los ciclos de estudio de la carrera de Educación Inicial en la UNAE. 
Gráfica 1. Elementos curriculares que orientan la formación docente en la carrera de Educación Inicial en la UNAE.

\begin{tabular}{|c|c|c|c|}
\hline & & Problema & PROYECTO DE INTEGRACIÓN DE SABERES (PIENSA) \\
\hline \multirow{4}{*}{ 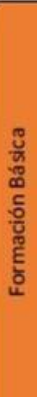 } & 1 & \multirow[t]{2}{*}{$\begin{array}{l}\text { ¿Qué sujetos, contextos y } \\
\text { sistemas socio-educativos? }\end{array}$} & $\begin{array}{l}\text { aproximación a la política pública en educación y del buen vivir (problemas de las instituciones de } \\
\text { educación en las dimensiones de organización académica e infraestructura) }\end{array}$ \\
\hline & II & & $\begin{array}{l}\text { contextos familiares-comunitarios y aprendizaje de los sujetos educativos (aproximación diagnóstica de } \\
\text { tensiones que presentan los aprendizajes de grupos especificos de estudiantes, relacionados con } \\
\text { contextos de socialización en sus dimensiones familiares y comunitarias: historias de vida) }\end{array}$ \\
\hline & III & \multirow[t]{2}{*}{ ¿Qué y cómo enseñar? } & $\begin{array}{l}\text { modelos pedagógicos aplicados en instituciones especificas (diseño y desarrollo de metodologías, } \\
\text { medios, trayectorias y valores de aprendizaje) }\end{array}$ \\
\hline & IV & & $\begin{array}{l}\text { modelos curriculares contextualizados y adaptados a los sujetos educativos aplicados en instituciones } \\
\text { especificas: diseño y desarrollo de proyectos currriculares cuya organización del proceso de } \\
\text { enseñanza-aprendizaje es contextualizada, flexible y adaptada a los sujetos educativos }\end{array}$ \\
\hline \multirow{3}{*}{ 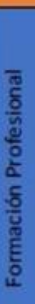 } & V & \multirow[t]{3}{*}{$\begin{array}{l}\text { ¿Qué ambientes, procesos } \\
\text { y resultados de aprendizaje }\end{array}$} & $\begin{array}{l}\text { diseño y construcción de escenarios, contextos y ambientes de aprendizaje (convergencia de medios y } \\
\text { aula invertida) }\end{array}$ \\
\hline & $\mathrm{VI}$ & & $\begin{array}{l}\text { diseño, aplicación y evaluación de recursos y estrategias educativas para la adaptación, flexibilización e } \\
\text { integralidad de experiencias de aprendizaje personalizados }\end{array}$ \\
\hline & \begin{tabular}{|l|} 
VII \\
\end{tabular} & & $\begin{array}{l}\text { diseño, aplicación y evaluación de modelos pedagógicos y curriculares adaptados a las necesidades de } \\
\text { aprendizaje(ritmos y estilos de aprendizaje; capacidades diversas) y culturales (integraciones históricas } \\
\text { y socio-culturales) }\end{array}$ \\
\hline \multirow{2}{*}{$\begin{array}{l}\frac{c}{\mathrm{O}} \\
\frac{\mathrm{D}}{\mathrm{D}} \\
\text { 른 }\end{array}$} & VIII & $\begin{array}{l}\text { ¿Qué valores y } \\
\text { mecanismos de } \\
\text { participación de los sujetos } \\
\text { que aprenden y de la } \\
\text { comunidad? }\end{array}$ & $\begin{array}{l}\text { diseño, aplicación y evaluación de modelos de intervención educativa comunitaria (interacciones } \\
\text { escuela-familia-comunidad) }\end{array}$ \\
\hline & IX & $\begin{array}{l}\text { ¿Qué funciones y perfil } \\
\text { docente? }\end{array}$ & $\begin{array}{l}\text { sistematización de la práctica de investigación-intervención educativa: elaboración del proyecto de } \\
\text { mejoramiento de contextos educativos redacción del informe final }\end{array}$ \\
\hline
\end{tabular}

Fuente: Las Autoras (2017).

A la luz del análisis del panorama de formación docente descrito que proyecta el carácter socio-comunitario que debe caracterizar el accionar preprofesional de los aprendices en los diferentes ciclos de estudio de la carrera de Educación Inicial en la UNAE, además de enfatizar en su perfil de docente-investigador mediante el desarrollo de las competencias profesionales afines y pertinentes que le permite emanciparse del accionar profesional de un actor educativo transformador de contextos educativos con compromiso social los docentes que imparten dicha formación en la UNAE.

El abordaje analítico de los procesos de la formación docente, desde y 
para escenarios socio-comunitarios en la referida carrera, bajo principios de RS que se derivan de manera particular sobre la base de la vinculación con la colectividad, constituyen los fundamentos esenciales de la modelación socio comunitaria formativa, proceso denominado de esta manera por la autoras para hacer referencia al carácter social y comunitario de la formación docente en la carrera de Educación Inicial, y al que deben tributar los procesos docentes e investigativos para perfilar el pensamiento teórico y práctico del estudiante en formación como docente-investigador con compromiso social.

La formación en Educación Inicial se inscribe en el modelo pedagógico institucional de la UNAE (2017b): que destaca "el compromiso y la implicación activa del docente... e incluye aspectos racionales y emotivos, conocimiento explícito y conocimiento tácito, técnicas y habilidades concretas y estrategias y modelos teóricos" (pág. 15).

La estructura y diseño de la malla curricular y el micro currículo de la carrera de Educación Inicial, invita a observar, reflexionar, analizar y sistematizar experiencias de enseñanza-aprendizaje (procesos de reflexión de las experiencias educativas). Concretar propuestas específicas de evaluación y valoración de procesos de enseñanza-aprendizaje, diseñar y validar propuestas de innovación educativa implementadas en la práctica integral, para teorizar la práctica y experimentar la teoría.

De tal forma, que la relación de la trilogía docencia, investigación y vinculación universitaria inicia su concreción desde el primer ciclo, se proporcionan oportunidades de enseñanza-aprendizaje marcadas por un compromiso educativo, participativo y socio-comunitario en el contexto intercultural ecuatoriano, mediante la vinculación con instituciones específicas de Educación Inicial, para propiciar procesos de aprendizaje orientados por elementos del diseño curricular respectivo.

El sentido de la dinámica curricular de la carrera, derivado de los ejes transversales y nudos problémicos antes mencionados, refuerza la vinculación 
con la colectividad desde y para la formación preprofesional socio-comunitaria de los estudiantes. Al respecto, el Modelo de Vinculación con la Sociedad (UNAE, s.f.), plantea que las prácticas preprofesionales son el "escenario clave para la identificación de necesidades de la comunidad, colaborando a la vez con el desarrollo de competencias socio-educativas en nuestros estudiantes por medio de la docencia, la innovación social y la investigación, mediante la práctica comunitaria" (pág. 11).

En consonancia a las aspiraciones de la RSU antes mencionadas, el proceso de desarrollo profesional de la carrera de Educación Inicial de la UNAE, mediante el voluntariado y el cumplimiento de las horas de vinculación con la colectividad propicia la articulación entre docencia-investigación y vinculación con la colectividad en procesos de aprendizaje de transformación en contextos reales, elemento que particulariza la formación UNAE.

\subsection{Avances y desafíos de la formación docente socio comunitaria en la carrera de Educación Inicial de la UNAE}

En consideración a los fundamentos planteados y del análisis retrospectivo de las autoras como docentes-investigadoras de la UNAE en el campo de formación de la praxis preprofesional en la carrera de Educación Inicial, se enfatiza en los rasgos y avances del escenario pedagógico-curricular de la UNAE que particularizan al carácter socio comunitario de la formación docente; así también, permite derivar en una caracterización de la formación del docente-investigador con compromiso social:

- La concepción pedagógica y curricular de la UNAE y su dinámica de aprendizajes, tributa a las aspiraciones de transformación de bienestar común, enmarcado en un enfoque sostenible y sobre la base de una comunicación multicultural entre los actores y agencias del territorio.

- Entre los principios pedagógicos-curriculares que permea la dinámica 
de formación docente con carácter socio comunitario, son desarrolladas las competencias específicas para perfilar la actuación preprofesional con compromiso social de los estudiantes, según lo establecido por la UNAE (2016b), una formación docente basada en la "filosofía pedagógica denominada aprendizaje y servicio que pretende integrar y enriquecer el aprendizaje profesional más relevante con el servicio a la comunidad en el ámbito educativo, atendiendo a las necesidades más prioritarias de la comunidad social" (pág. 22).

- Actuación pertinente de los actores internos de la UNAE para tributar desde un enfoque integrador y sistémico a los concepciones, acciones y prácticas que perfilan la formación docente desde y para lo sociocomunitario. Los procesos de formación docente de la carrera de Educación Inicial demanda por parte de los formadores de formadores en sus procesos de docencia e investigación, además de las instancias de gestión respectiva, un singular énfasis en los escenarios de aprendizaje y de investigación formativa, relacionado a la pertinencia socio-comunitaria de la formación teórica, práctica y metodológica para la modelación del perfil del futuro docente-investigador.

- La modelación socio comunitaria formativa en la Educación Inicial de la UNAE se concreta mediante la teorización de la práctica socioeducativa y la experimentación de la teoría inherente para derivar en el compromiso social de la labor docente, mediante el desarrollo de las competencias profesionales, según las particularidades e identidad de la carrera de Educación Inicial.

El redimensionamiento de la función de la vinculación con la colectividad como el escenario para el compromiso con la realidad social, desde el protocolo institucional que contiene las aspiraciones al respecto, 
hasta explicitarla en el acto educativo transformador, es decir en los procesos del ejercicio de la profesión docente de sus actores, mediante una dinámica integradora entre la docencia y la investigación, sobre la base de los fundamentos políticos, sociales, epistemológicos, pedagógicos y curriculares que lo conforman. Al respecto, se asume lo planteado por Rojas (2018):

La responsabilidad ética-social en y en la comunidad inmersa en el entorno para un proceso imaginado en la orientación hacia la construcción de aprendizajes desde un enfoque constructivista, lo que permite, en este momento una manera diferente de pensamiento y mediación de epistemes ante la complejidad de los contextos, inherentes a la educación universitaria (pág. 313).

En tal sentido, emerge como aporte esencial del presente análisis que los procesos pedagógicos para la modelación teórica, metodológica y práctica socio-comunitaria en la formación docente se conciban por parte de los actores de la gestión académica y pedagógica de manera explícita, intencionada, programada y concreta desde la planificación de los procesos de gestión de los aprendizajes; prioritariamente en las asignaturas de la praxis preprofesional (Cátedra Integradora y Prácticas); así mismo y en este sentido que tributen a dicho fin, la dinámica inter y transdisciplinaria de las asignaturas del campo de formación teórico y metodológico de la carrera. De esta manera las actividades de aprendizaje se llevarían a cabo de formación pertinente, destinadas al logro de saberes, conocimientos, habilidades, actitudes y valores vinculados al compromiso social y responsabilidad que caracteriza a un docente investigador idóneo para la Educación Inicial.

Por lo expuesto anteriormente, el perfil de docente-investigador de la UNAE, responde a la formación de actores que se comprometan con la transformación del sistema educativo nacional que además de atender y potencializar las capacidades de niños y niñas entre 0 a 5 años de edad, los reconozcan como sujetos partícipes de la vida colectiva, en el contexto 
ecuatoriano, latinoamericano e internacional.

El referido perfil profesional, se orienta hacia el desarrollo de competencias profesionales, investigativas, docentes y de gestión pedagógica y socio-comunitaria, que promuevan la democratización de la vida cotidiana de los niños y las niñas, propiciando ambientes educativos emancipadores, sobre la base sinérgica de la escuela, la familia y la comunidad. Se busca, de esta manera, formar docentes con capacidad para analizar críticamente los escenarios educativos y socio-comunitarios en conexión con los estudios y bases conceptuales de la pedagogía y la investigación educativa.

En cuanto a los desafíos que emergen del contexto del presente análisis para la formación docente socio-comunitaria desde el accionar de la carrera de Educación Inicial en la UNAE, se pueden precisar los siguientes:

- Explicitar en los protocolos de la planificación estratégica institucional, las agendas, programas y sílabos de la referida carrera, los alcances de los aprendizajes con pertinencia educativa y socio-comunitaria.

- Promover de manera permanente y sistemática procesos que faciliten la concienciación de los actores educativos en cuanto al carácter sociocomunitario de la formación docente, en el campo teórico, metodológico y práctico, desde y para los programas y proyectos de vinculación con la sociedad concebidos para la carrera de Educación Inicial.

- Propiciar el intercambio de experiencias docentes, investigativas y de proyectos de vinculación con la colectividad en cuanto a su alcance y carácter transformador en el escenario de las prácticas preprofesionales y comunitaria, además de su trascendencia en territorio mediante indicadores de resultados e impacto.

- Construcción de las competencias profesionales de carácter sociocomunitario que desarrollan los formadores de formadores y los 
estudiantes en formación docente; considerando los ejes de la práctica preprofesional y los principios de la vinculación con la sociedad.

- Profundizar en los fundamentos teóricos-metodológicos de la modelación socio-comunitaria formativa en la carrera Educación Inicial de la UNAE como una categoría teórica, metodológica y práctica de la formación docente del futuro egresado.

- Mantener una revisión permanente de los elementos esenciales de la formación teórica, metodológica y práctica de índole socio-comunitaria para redimensionamiento del perfil egresado.

\section{Conclusiones}

La UNAE responde a una necesidad de transformar la educación desde una perspectiva liberadora y crítica. En particular, la universidad se piensa desde una iniciativa fundamentada en la igualdad, el derecho a una educación gratuita y de calidad, la democratización del aula, la Gestión Social del Conocimiento y el fomento de la investigación y el bienestar colectivo. Esta reflexión permite generar un análisis desde la carrera de Educación Inicial sobre la formación docente socio comunitaria, escenario donde los actores educativos y comunitarios, no son concebidos como objetos/sujetos de estudio, sino como co-creadores de conocimientos y saberes.

La construcción de la fundamentación y caracterización de la formación docente de la carrera de Educación Inicial en el contexto de la UNAE, desde y para un enfoque socio comunitario permitió precisar relaciones no explicitadas en este sentido, desde y para la dimensión pedagógica, curricular y práctica para derivar en el redimensionamiento de dicha formación, sobre la mejora de la relación "simbiótica" entre la Universidad y el territorio, desde un accionar sistemático, intencionado y planificado de la gestión de los aprendizajes, bajo una mirada crítica, reflexiva, consensuada y de construcción mutua de carácter 
sostenible y dinamizado por las prácticas preprofesionales.

El perfil profesional del docente investigador con pertinencia social, debe responder a las particularidades e identidad de la referida carrera. Las competencias profesionales e investigativas específicas, se derivan de las necesidades y demandas del territorio, construyendo y re-construyendo una dinámica de formación y acción "simbiótica" o "asociativa" entre el contexto universitario, las instituciones educativas y sus respectivas comunidades. La UNAE ante el desafío de cumplir con RSU encomendada y de garantizar egresados con una formación docente socio comunitaria de calidad ante la demanda de la Educación Inicial del Ecuador; el territorio, constituido por las instituciones educativas específicas de Educación Inicial donde se realizan las prácticas preprofesionales, un sistema humano y social complejo, demandante de acciones y atención a situaciones o problemas socio-educativos reales.

Para tal fin urge asumir los desafíos institucionales y pedagógicos, concretar escenarios de aprendizaje desde y para una praxis profesional con sentido y pertinencia social, sobre la teorización de la práctica socio-educativa y la experimentación de la teoría inherente para brindar condiciones para el tránsito del conocimiento planteado por Santos, desde los "conocimientos universitarios" pedagógicos y científicos a "conocimientos pluriuniversitarios" que aporten a la solución de problemas socio-educativos.

\section{Referencias}

Asamblea Nacional Constituyente (2008). Constitución de la República del

Ecuador. Registro Oficial 449 de 20-oct-2008. Ecuador: Montecristi.

CES (2016). Reglamento de Régimen Académico. Quito, Ecuador: Consejo de Educación Superior.

Herdoiza, M. (2015). Construyendo igualdad en la educación superior. 1 ra. ed. Quito, Cuenca: Senescyt/Unesco.

Martínez, C. (2011). Responsabilidad Social Universitaria y su 
Articulación con las Funciones Docencia-Investigación-Extensión para su Vinculación con el Entorno Social. Boletín IESALC Informa de Educación Superior. Nro. 214. Venezuela: UNESCO IESALC.

PNUD (2016). Objetivos de Desarrollo Sostenible. Nueva York, Estados Unidos: Programa de las Naciones Unidas para el Desarrollo. Recuperado de:

http://www.undp.org/content/undp/es/home/sustainable-developmentgoals.html

Rojas, M. (2018). Nociones Críticas en la Responsabilidad Social de la Extensión Universitaria con la Vinculación Comunidad. Revista Scientific, 3(10), 304-316. Recuperado de:

https://doi.org/10.29394/Scientific.issn.2542-2987.2018.3.10.17.304$\underline{316}$

Santos, B. (2004). La Universidad en el Siglo XXI. Para una reforma democrática y emancipadora de la Universidad. Buenos Aires, Argentina: Miño y Dávila.

UNAE (2018). Modelo de prácticas preprofesionales de la UNAE. Azogues, Ecuador: Universidad Nacional de Educación - UNAE.

UNAE (2017a,b). Modelo Pedagógico de la UNAE. Azogues, Cuenca: Universidad Nacional de Educación - UNAE.

UNAE (2016a,b). Estatuto de la Universidad Nacional de Educación. Azogues, Ecuador: Universidad Nacional de Educación - UNAE.

UNAE (s.f.). Modelo de vinculación con la colectividad de la UNAE. Azogues, Ecuador: Universidad Nacional de Educación - UNAE.

Vallaeys, F. (2014). La responsabilidad social universitaria: un nuevo modelo universitario contra la mercantilización. Revista iberoamericana de educación superior 5(12), 105-117, ISSN: 20072872. Recuperado de:

https://www.redalyc.org/pdf/2991/299129977006.pdf 


\section{Ormary Egleé Barberi Ruiz \\ e-mail: ormary.barberi@unae.edu.ec}

Nacida en Caracas, Venezuela. Doctora en Ciencias Pedagógicas, Especialización en Desarrollo Comunitario. Experiencia profesional en docencia e investigación en educación inicial, básica y superior; dirección y supervisión de instituciones educativas y administrativas del sistema educativo venezolano. Docente de pregrado y postgrado en la Universidad Pedagógica Experimental Libertador-UPEL y Universidad Nacional de los Llanos Occidentales Ezequiel Zamora-UNELLEZ. Ponente en eventos nacionales e internacionales y autora de artículos regionales e internacionales con énfasis en temas de gestión escolar, pedagogía, didáctica, praxis profesional y formación docente. Actualmente, se desempeña como docente-investigadora en la Universidad Nacional de Educación (UNAE) en Ecuador. 


\section{Gisselle Margarita Tur Porres}

e-mail: gisselle.tur@unae.edu.ec

Nacida en Montevideo, Uruguay. Doctora en Ciencias

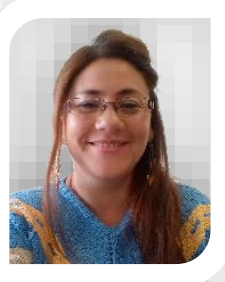
de la Educación, con experiencia en coordinación y gestión de fondos de proyectos de educación, investigación e innovación, nacionales e internacionales. Experiencia docente y de investigación en educación inicial, básica y superior, en programas, proyectos formales y no formales, con niños, adolescentes y familias en situación de vulnerabilidad, en Bélgica, Ecuador y Uruguay. Actualmente, se desempeña como Coordinadora Pedagógica de la Universidad Nacional de Educación, donde anteriormente, estuvo a cargo de la Dirección de la Licenciatura en Ciencias de la Educación Inicial. 